\title{
Smart Identification System on Internet of Things for User Authentication
}

\author{
Antonidoss $\mathrm{A}^{1}$, Bhavani $\mathrm{R}^{2}$, Prabha $\mathrm{M}^{3}$, Manju $\mathrm{S}^{4}$, Chinnadurai $\mathrm{G}^{5}$ \\ \{ aro.antoni@gmail.com ${ }^{1}$, bhavaniramamurthi@gmail.com², prabha110292@gmail.com ${ }^{3}$, \\ smanju1102@gmail.com ${ }^{4}$,china_durai@yahoo.co.in $\left.{ }^{5}\right\}$
}

Associate Professor, Department of Computer Science and Engineering, Hindustan Institute of Technology and Science, Chennai ${ }^{1}$, Assistant Professor, Department of Information Technology, St.Joseph college of Engineering, Chennai ${ }^{2}$, Assistant Professor, Department of Electronics and Communication Engineering, St.Joseph college of Engineering, Chennai ${ }^{3}$, Assistant Professor, Department of Electronics and Communication Engineering, St.Joseph college of Engineering, Chennai ${ }^{4}$, Assistant Professor, Department of Electronics and Communication Engineering, Rajiv Gandhi College of Engineering, Chennai ${ }^{5}$.

\begin{abstract}
Regarding that, clients agreed on the value of reliable Internet access regardless of location, mode, or time. Individuals can now access a few top-tier administrations through the Internet of Things (IoT), which is a heterogeneous network characterized by machine-to-machine communication. Regardless of how the devices are used to set up the communications, the clients should be considered the true producers of data and purchasers of the yield data. As a result, clients should be viewed as a critical component of IoT; as a result, client distinguishing evidence, verification, and approval are required. In any case, the client Index measure is too perplexing, given the clients' apprehension about sharing their confidential and private information. However, some of their devices should be able to access this personal information. Appropriately, an unbiased method for understanding clients and coping with their personalities is important. Furthermore, the customer plays a minor but significant role in laying the groundwork for the character distinguishing proof and ensuring the consistency of open services. The main goal of this paper is to create a new identity management system (IdMS) for the Internet of Things. The main commitments of this paper are the suggestion of a gadget acknowledgment calculation for client identifiable evidence, the suggestion of a different identifier configuration, and a hypothetical IdMS framework.
\end{abstract}

Keywords: Authentication, identification algorithm, identity management, internet of things.

\section{Introduction}

The web is the solitary interlinked framework that permits worldwide correspondence between gadgets through a few standard conventions and associations of various kinds of organizations (government, business, scholarly, etc.,). In the first place, the web permitted correspondence through messages and addressed as static sites. Be that as it may, these days, there are numerous executions of the web, which is noticed anyplace in a few types of life as a bunch of gave applications and administrations by meeting clients' prerequisites paying little heed to the area and the time. This is an immediate result of the client and components digitalization [1]. 
The web innovation request is reflected in every gadget of clients. It is getting convenient and drawing near to the client more than everything and like never before. These days, savvy gadgets give a consistent overall association, and this association gets necessary in ordinary lives. On account of the expanding number of associated gadgets, there is a need of an instrument permitting self-governing correspondence between gadgets [2]. The IoT is considered as an empowering arrangement. IoT is an organization permitting direct association between gadgets utilizing a novel identifier for looking for data. The yield of Machine-to-Machine (M2M) correspondence compares/created to/by clients [3]. Also, the clients are the owner of data; in this manner, client distinguishing proof and confirmation, secure correspondence foundation, and assets access are fundamental.

The clients address a fundamental part of the Internet of Things (IoT), and hence, they are viewed as shrewd things that make, assemble and oversee data utilizing individual or/and normal gadgets, and subsequently, clients ought to be recognized in the IoT likewise like different things (gadget, sensor, and actuator). Clients are firmly engaged with IoT since they influence the present ubiquitous web, and they make electronic gadgets and make more fitting UIs. Since the client is a vital part, his ID is obligatory. This addresses an alluring region for examination to discover answers for personality the board frameworks that save exertion and time.

Actually, the IoT is an arrangement of a limitless arrangement of connected things (like actuators, sensors, gadgets) that offer a few types of assistance through the web. Therefore, IoT addresses another chance for business, gadgets' execution, prompting administrations for clients. The presence of a few designs and conventions for sensors and gadgets connection in IoT and the absence of uniform arrangement, show the need for overseeing characters to guarantee the achievement of things association model [4]. As per various proposed models, client ID and verification are reliant on network geography, offices, and guidelines [5].

To resolve this problem, this paper proposes a new IdMS. By perceiving items in IoT, the proposed model means to separate clients and have client-focused administrations. Singular Thing Sign-On is a feature of the IdMS. The proposed model limits the scope of the inquiry to IoT and M2M.

It focuses in particular on the IdMS, which considers all aspects of IoT. (for instance, individuals, gadgets, and non-human interface gadgets like sensors and actuators). The distinguishing proof and evidence mechanisms, as well as its inquiries and recommendations, are depicted.

We also look at the client ID issues and suggest a new design for the Single Thing SignOn Identity Management System (IdMS), which is focused on the end-user and is a clientarranged support framework. By distinguishing only one of the client's objects, the recommended design allows perceiving the client and his appointed administrations (gadget, sensor, etc.,). In addition, to differentiate the client, we suggest a calculation called Device Recognition (DR). To illustrate the concept, the DR measurement is hypothetically evaluated. The results support the relevance of the research issue.

The IdMS has finally been depicted. The IdMS requirements (client and framework requirements) are highlighted in particular. As a result, a new IdMS method is proposed that deals with the characteristics of items. 


\section{Analysis of Identity}

\subsection{Recognization}

In today's world, personality is the window through which a client can communicate with his or her articles and harassment administrations. The personality definition is extended to things in the IoT's particular circumstance. Personality is regarded as an endpoint that allows concerned things to openly and effectively access endpoints [6]. Clients can use and change information through the well-known proof cycle, which also allows for customization of administrations and communications [7]. ID in IoT partners assigns an identifier to an address as appropriate. The quality of a thing, such as sensors with the name Radio Frequency Identifier, is an unmistakable property. The application space relies on the identifier, which distinguishes one thing from another. Since the identifier's sole purpose is to view items in a unique way, it should be strong. The frail identifier gives various items in the system its motivation [8]. The IdMS is in charge of creating, managing, and receiving personalities [9].

\subsection{Authentication}

Validation is known as the creation of a personality between related items (clients or gadgets). Because of the variety of items, there is a criterion for attack resistance and a minor response for verification. The client and gadget validation are each depicted in detail in the following sections.

Client Authentication: The testing period approves the client's displayed personality to see whether it is true or not by referencing accreditations. The word "certification" refers to confirmation device or identity verification. It is a test or validation measure stage that aids in the confirmation of the client's identity in relation to the system ID (for example, network address). The certification is crucial for validation since it displays a snippet of data (secret word) or specific properties (such as NFC and RFID labels or voice/face recognition). Confirmation can include one or more certifications, as well as accreditation [10]:

- Something is gained: the client hands over an unmistakable item containing the client's secret data, which is necessary for the confirmation contact. A USB stick, a brilliant card, or any unmistakable item may be used. Since the secret data is known for the secret word, there is no compelling reason to remember it. However, since clients can exchange articles and objects can be misplaced or stolen, how can you ensure that it gives the correct client ID [11].

- Possession of something:The client provides biometric data, which includes specific physical and social properties such as speech, advanced picture of the face, retina, unique mark, and so on. Despite the fact that biometric data is new and intended to be unaltered, there is a risk associated with its intentional or unintentional use (taken, duplicated, or misrepresented) [6].

- Something that is well known: The client provides confidential information (for example, username/secret key, designs, and graphical images). These techniques allow the client to remember private data, which is usually difficult to keep hidden from other clients [6].

\subsection{Accounting and Authorization}

The approval period point is to verify whether a specific client has the option to use a specific asset (information or gadget), while the verification interaction point is to validate client personality. The approval period is carried out in different locations depending on the security strategy (i.e., there is a comparison between the validated thing (for example, asset access) authorizations and the asset security strategy [12]. 
There can be four different types of user access techniques available.

- Quality Based Access Control: The character credits (rather than the actual personality) give the way to permitting admittance to a specific asset. Consequently, this method can't recognize a particular personality. To build the security viewpoint, all things, including clients, exercises are archived and saved. The methodology is named bookkeeping, and from a security point of view, it is compelling since it is executed paying little mind to the achievement or the disappointment of the confirmation interaction, and it is considered as evidence if there should arise an occurrence of a security examination.

- Discretionary and Obligatory Access Control: The consents provider is the point of convergence here. In most cases, an important executive determines the authorizations for each framework asset in mandatory access control. Regardless, in discretionary access control, the client establishes asset entry authorizations by contacting the asset's owner.

- Work Based Access Control: A new layer called the job layer is introduced to deal with the consents mission, and the jobs are considered authorization subsets. The permission to enter is linked to the work rather than a particular asset. As a result, the asset has a variety of jobs that allow it to operate based on a few authorization subsets.

- Access Control Lists: this strategy characterizes a rundown of consents doled out to an asset. This strategy figures out which clients are approved to admittance to assets, just as what activities are allowed on specific assets. By and large, an entrance control framework addresses a steady method to proclaim access authorizations in a network characterizing things-assets consents. The primary impediment of this methodology is the intricacy of dealing with a colossal number of assets and things.

\section{Smart Identification System}

This segment depicts the proposed Smart Identification System (SIS) for recognizing the client. It worth referencing that the proposed SIS calculation doesn't dispose of the most mainstream and notable ID strategy: the username and secret key, yet it very well may be seen as a strengthening technique to mechanize (without client intercession) the verification interaction, nonetheless, the manual validation is still alternatively utilized. Figure 1 presents the SIS calculation.

Before all else, the calculation appoints for every client a Smart Sheet (SA). SA is a rundown of distinguished gadgets of client A. Note that SA is novel. Every gadget D is put away in SA list with an extraordinary number inside $[1, . ., \mathrm{m}]$ (to show there are $\mathrm{m}$ kinds of gadgets). Likewise, for every gadget type Dm there is a bunch of unmistakable identifiers types id, which is put away in SA list, with a one of a kind number inside $[1, . ., \mathrm{n}]$. Consequently, all gadget character sorts of the client (IdnDm) are recorded in SA list.

At whatever point a recognizable proof solicitation is gotten from one of the recorded gadgets in a particular space, a programmed search begins to distinguish other client gadgets and to check the quantity of accessible client gadgets in the neighborhood area. For this situation, the client can determine the security level by taking care of the quantity of gadgets needed as client character confirmation. For example, the client recommends the recognizable proof of 2 out of 4 individual gadgets all the while to be a standard for his programmed 
personality ID. Since the client is a piece of IoT, he is considered as a guidelines chief in the framework, concerning his inclinations.

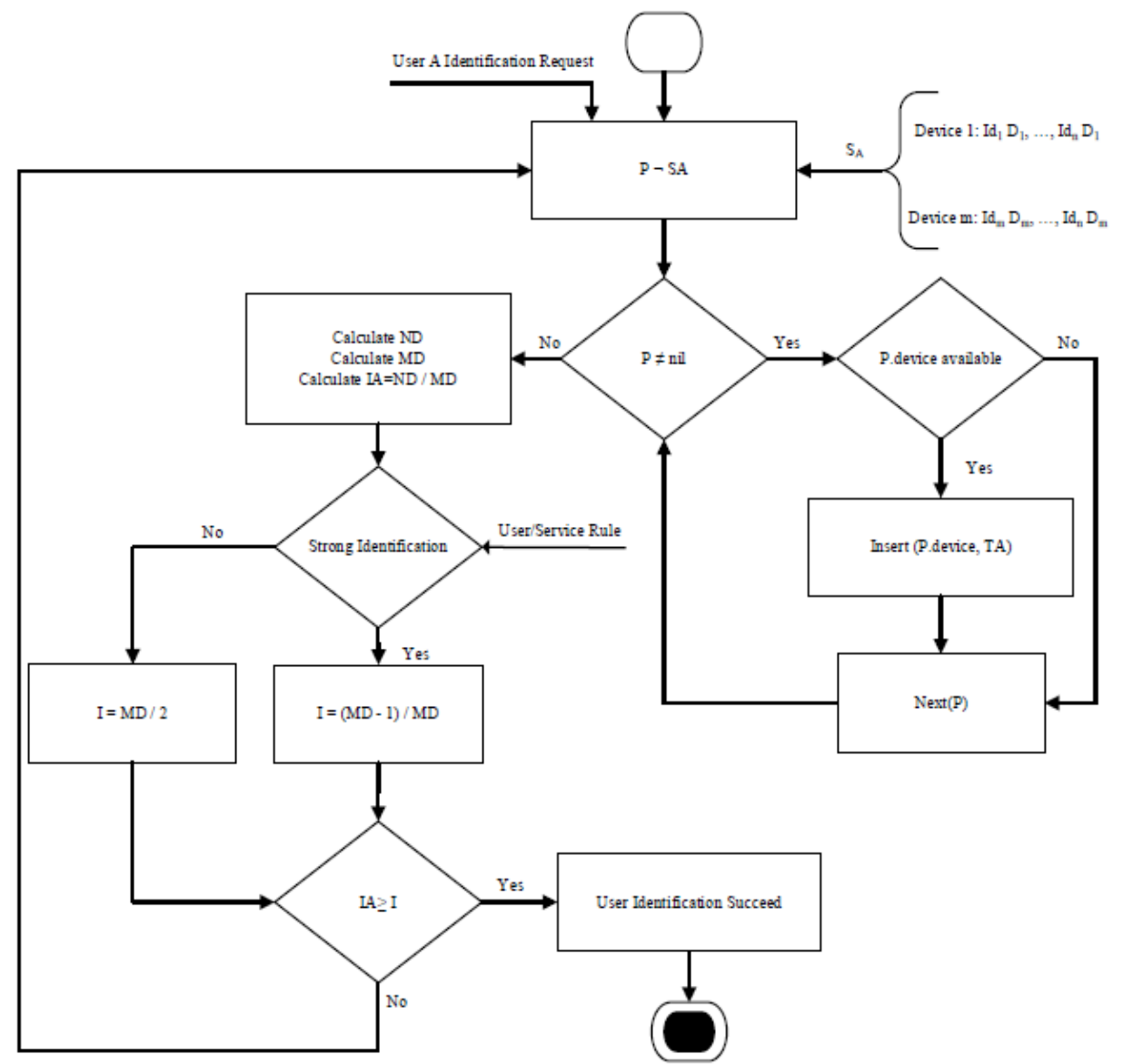

Fig.1. Smart Identification calculation

At that point, the calculation identifies and checks the quantity of accessible gadgets of client An and recorded in TA' sheet. From that point, the calculation ascertains the IA list addressing the proportion between the quantity of gadgets in SA sheet and the quantity of gadgets in TA sheet at a given time. At last, the calculation checks the required distinguishing proof level. In the present circumstance, there are two potential cases: solid and frail ID dependent on rules of client or administrations.

- Solid distinguishing proof: This record (addressing Index of Strong ID) asserts that all client gadgets ought to be accessible (identified and perceived) aside from one. In this way, the calculation analyzes the IDR list to IST record. In the event that I $\geq$ IST, the client recognizable proof succeeds. Something else, the calculation is executed iteratively until the assistance break ends.

- Powerless ID: The IWK file (addressing Index of Weak ID) asserts that at any rate half of the client gadgets ought to be accessible (identified and perceived). Hence, the calculation analyzes the IDR file to IWK list. Assuming I $\geq$ IWK, the client 
recognizable proof succeeds. Something else, the calculation is executed iteratively until the assistance break ends.

Appropriately, the recognizable proof rate addressed by Equation (1) is utilized to evaluate the Smart Identification calculation.

$$
\mathrm{IDR}=\mathrm{NG} / \mathrm{MG}
$$

Where, IDR: characterizes the coefficient addressing the recognizable proof rate.

NG: characterizes the quantity of distinguished gadgets related with a particular client. Officially, NG is the quantity of gadgets in TA' sheet.

MG: characterizes the quantity of whole predefined gadgets needed for client ID. Officially, MG is the quantity of gadgets in SA' sheet.

Since the ID coefficient IDR of Smart Identification figuring depend on the quantity of perceived client gadgets, it is required that this coefficient should more like '1' to distinguish the client himself.

\section{Smart Identification calculation Analysis}

As referenced already, the Smart Identification computation doesn't reject the normal and mainstream login strategies. Nonetheless, the calculation is new, and it saves exertion and time since it presents a modernized technique for client distinguishing proof and validation. In addition, the shortfall of some client gadgets (lost or taken) permits the disappointment of the distinguishing proof and confirmation measures. Thusly, the admittance to the client's private data or administrations will be unapproved, on the other hand to the secret phrase saved money on the gadget. As an insurance against the non-accessibility of some client's gadgets at the confirmation time, the Smart Identification figuring permits an elective recognizable proof by entering the secret phrase (predefined by the client) physically secret word passage. In this way, the calculation permits the client's recognizable proof taking all things together cases. The recommended ID rate I see Equation (1) permits programmed client distinguishing proof and validation.

\section{Heterogeneous IoT Networks Recognition Schema}

The heterogeneous organizations and structures are talked about in this part. It begins by summing up the proposed ID plans in the writing by posting their benefits and weaknesses. In this manner, we recognize research difficulties. Along these lines, we acquaint another identifier design with meet the goal of a solid answer for personality the executives. At last, we assess the recommended identifier design.

\subsection{Heterogeneous Internet of Things Networks}

The Internet of Things (IoT) is a series of various interconnected things (mechanical or advanced), actuators, sensors, or only things that obey the trademark "all can be linked to the Web." The Internet of Things (IoT) provides an eco-system of services and smart programming that are used to improve and streamline human life and daily tasks. The Internet of Things (IoT) is a strongly linked development to M2M. IoT is placed as a framework of establishing and supporting M2M connections [13]. In [14], the nuances of M2M engineering are discussed.

5.2 Identification Format Formulated 
A pre-owned identifier incorporates the total data of the personality for something specific. All in all, the identifier characterizes the space, client, his gadget, and non-UI gadget remarkably. This identifier doles out the owner to things as proposed in [15]. Thing in IoT addresses clients, data, or gadgets; in this manner, it is obligatory to realize the thing ascribes. Everything is related with a novel identifier and set of traits.

Our recommended identifier design, which consolidates halfway identifiers, is:

Gadgettype\|GDinterface $\|$ LKinterface $\|$ DomainGId\|GadgetId\|ClientId

where,

- Gadgettype: demonstrates a fractional identifier that characterizes the sort of the gadget (for instance, human client, PC gadget, actuator, sensor, etc.,).

- GDinterface: demonstrates a fractional identifier that characterizes the worldwide interface or possession, and it is vital because of gadget versatility.

- LKinterface: demonstrates a fractional identifier that characterizes the neighborhood interface or proprietorship, and it is fundamental because of gadget locatio

- DomainGId: shows a fractional identifier that characterizes the area of thing enrollment, and it is essential because of the presence of certain spaces having a similar identifier yet enlisted in various Identity Providers.

- GadgetId: demonstrates a fractional identifier that characterizes an interesting identifier for every gadget.

- ClientId: demonstrates a fractional identifier that characterizes a special identifier for every client (gadget proprietor) in light of a specific area.

The suggested identification design aims to improve the client experience by allowing for programmed assisting that would be less complex and time-consuming than all other methods of client identification and association currently available. Despite the fact that the relationship seems normal and simple, it conceals a perplexing acknowledgement and reconciliation. Such complications were concealed, and the client's perspective is ignored.

\section{Framework for Managing Identity}

Identity Management System is characterized as a bunch of programming parts and coordinated hand-worked exercises. The reason for IdMS is the distinguishing proof and observing of processing assets usage and the help of information trustworthiness and security. Besides, IdMS includes numerous exercises, for example, produce testament, oversee trait and job, validate and control access, and so on IdMS encases a bunch of conveyed programming segments and a huge number of systems administration conventions. In addition, since the IdMS interfaces with business segments, its administration methodology should adjust to business morals, HR, and laws guidelines. Hence, IdMS plan and organization ought to consider the refered to standards to carry out effective IdMS. The associations between the IdMS administrations layer and things layers ought to be secure, and the entrance ought to be controlled.

\subsection{Architecture in IoT}

The whole article suggests IdMS engineering, which includes a single layer. A list of IoT strategies is included in the IdMS engineering. Figure 2 illustrates the IdMS engineering. IdMS examines a person's personality by examining their character and characteristics. We present a domain identifier (DomId) that is dependent on the application area and supports climate data. The suggested engineering's main benchmarks were linked to both the board's 
location, character affiliation and preparation, confirmation access and oversight, and, inevitably, deep rooted management using personalities and abilities as knowledge sources.
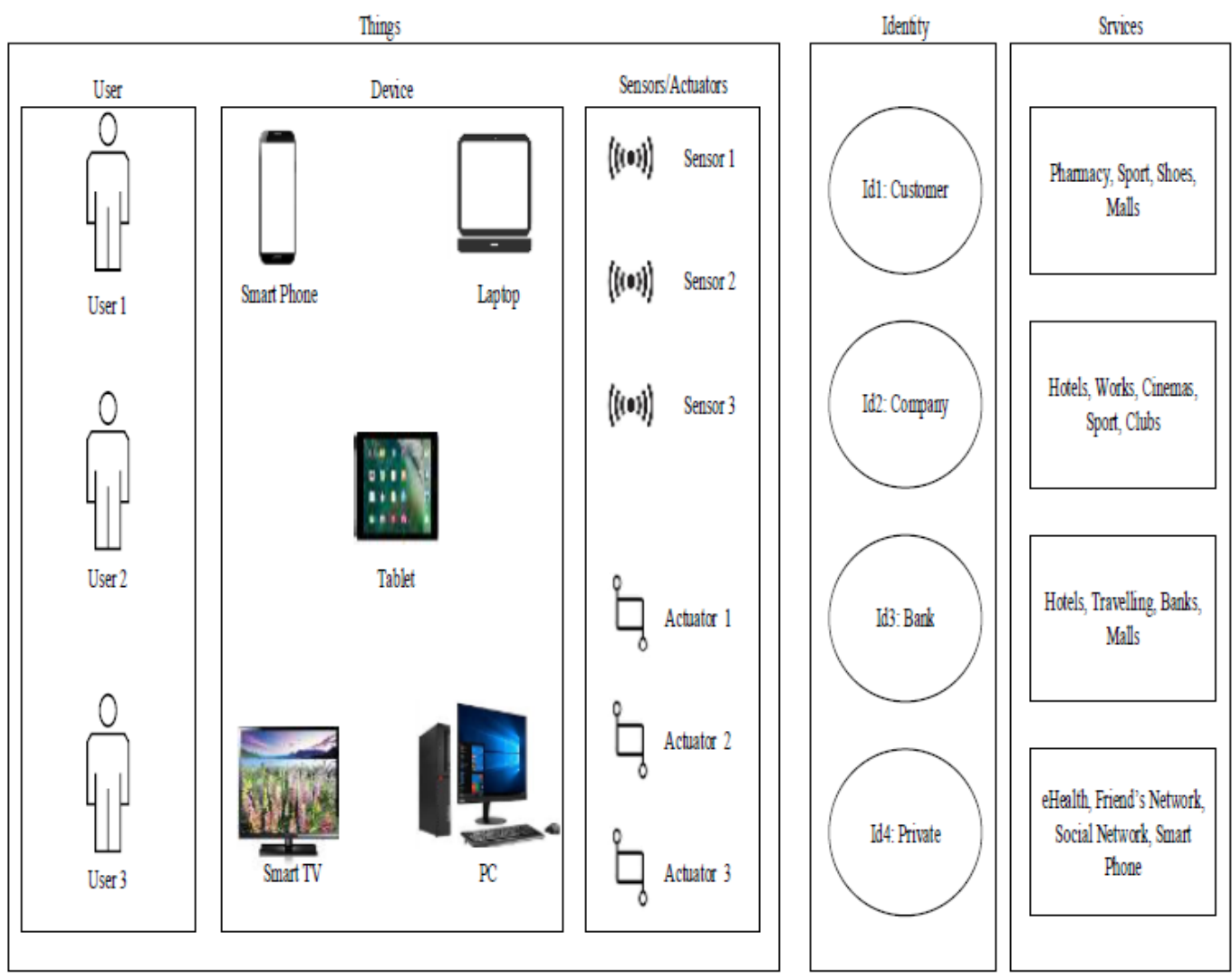

Fig. 2. IdMS architecture

In principle, the conceptual IdMS framework is yet another empowering engineering for handling characters in the Internet of Things. Nonetheless, for the assessment and review of IdMS proficiency, the execution of the IdMS model and its acceptance is critical. The proponents of the proposed IdMS lean on Information Communication Technologies to include storage space, operating procedures, clear model antiquities and administrations, and heterogeneous organization correspondence, among other things. As a result, a model for data connection and coordination among various members is important.

\section{Conclusions}

This paper defines the Internet of Things and describes the ID, validation, and acceptance cycles. It depicts the importance of smart IdMS implementation and suggests a fitting use case scenario to clarify and visualize the ID cycle's challenges. As a result, a Smart Identification System (SIS) calculation is proposed in order to identify gadgets in a timely and error-free manner. We suggested a factor that characterizes the gadget recognizable proof rate to test and examine the SIS estimate. 
The report specifically portrays heterogeneous organizations and their engineering in the following section. Similarly, we differentiate the review problems by addressing the familiar proof plans and identifier designs suggested in the writing. Finally, we'll show you another identifier design that helps to personalize board perspectives. There is a conversation more about suggested identity design's evaluation in terms of gadget form, gadget versatility, and system adaptability.

The study presented a new and broader perspective for characterizing that panel in IoT in order to allow modernized communication between different items in IoT while saving clients' time and effort.

\section{References}

[1] Baker S.A., Nori A.S. (2021) Internet of Things Security: A Survey. In: Anbar M., Abdullah N., Manickam S. (eds) Advances in Cyber Security. ACeS 2020. Communications in Computer and Information Science, vol 1347. Springer, Singapore. https://doi.org/10.1007/978-981-33-6835-4_7

[2] Wang W., Tao X. (2018) A User Identification Algorithm for High-Speed Rail Network Based on Switching Link. In: Li K., Li W., Chen Z., Liu Y. (eds) Computational Intelligence and Intelligent Systems. ISICA 2017. Communications in Computer and Information Science, vol 874. Springer, Singapore. https://doi.org/10.1007/978-981-13-1651-7_48

[3] Mnasri, W., Azaouzi, M. \& Romdhane, L.B. Parallel social behavior-based algorithm for identification of influential users in social network. Appl Intell (2021). https://doi.org/10.1007/s10489-021-02203-x

[4] H. Ning, Z. Zhen, F. Shi and M. Daneshmand, "A Survey of Identity Modeling and Identity Addressing in Internet of Things," in IEEE Internet of Things Journal, vol. 7, no. 6, pp. 46974710, June 2020, doi: 10.1109/JIOT.2020.2971773.

[5] D. Ghoshal, "A proposed technique of online face authentication to be used for the user identification," 2012 International Conference on Computer Communication and Informatics, Coimbatore, India, 2012, pp. 1-5, doi: 10.1109/ICCCI.2012.6158786.

[6] A. Theodouli, K. Moschou, K. Votis, D. Tzovaras, J. Lauinger and S. Steinhorst, "Towards a Blockchain-based Identity and Trust Management Framework for the IoV Ecosystem," 2020 Global Internet of Things Summit (GIoTS), Dublin, Ireland, 2020, pp. 1-6, doi: 10.1109/GIOTS49054.2020.9119623.

[7] Burmann C., Riley NM., Halaszovich T., Schade M. (2017) The Concept of Identity-Based Brand Management. In: Identity-Based Brand Management. Springer Gabler, Wiesbaden. https://doi.org/10.1007/978-3-658-13561-4_2

[8] G. Ben Ayed, "Digital Identity Metadata Scheme: A Technical Approach to Reduce Digital Identity Risks," 2011 IEEE Workshops of International Conference on Advanced Information Networking and Applications, Biopolis, Singapore, 2011, pp. 607-612, doi: 10.1109/WAINA.2011.118.

[9] H. Kim and E. A. Lee, "Authentication and Authorization for the Internet of Things," in IT Professional, vol. 19, no. 5, pp. 27-33, 2017, doi: 10.1109/MITP.2017.3680960.

[10] M. Shahzad and M. P. Singh, "Continuous Authentication and Authorization for the Internet of Things," in IEEE Internet Computing, vol. 21, no. 2, pp. 86-90, Mar.-Apr. 2017, doi: 10.1109/MIC.2017.33

[11] "IEEE Standard for Authentication in Host Attachments of Transient Storage Devices," in IEEE Std 1667-2009 (Revision of IEEE Std 1667-2006), vol., no., pp.1-125, 26 March 2010, doi: 10.1109/IEEESTD.2010.5438723.

[12] W. Lei and L. Xu, "Research and implementation of access control model of internet of things," 2016 5th International Conference on Computer Science and Network Technology (ICCSNT), Changchun, 2016, pp. 102-106, doi: 10.1109/ICCSNT.2016.8070128. 
[13] Song, J., Kunz, A., Schmidt, M. et al. Connecting and Managing M2M Devices in the Future Internet. Mobile Netw Appl19, 4-17 (2014). https://doi.org/10.1007/s11036-013-0480-9

[14] R. Zheng, Y. Zhao and B. Chen, "Device-Free and Robust User Identification in Smart Environment Using WiFi Signal," 2017 IEEE International Symposium on Parallel and Distributed Processing with Applications and 2017 IEEE International Conference on Ubiquitous Computing and Communications (ISPA/IUCC), Guangzhou, China, 2017, pp. 1039-1046, doi: 10.1109/ISPA/IUCC.2017.00158.

[15] S. Pal, M. Hitchens and V. Varadharajan, "Towards the Design of a Trust Management Framework for the Internet of Things," 2019 13th International Conference on Sensing Technology (ICST), Sydney, NSW, Australia, 2019, pp. 1-7, doi: 10.1109/ICST46873.2019.9047734. 